\title{
Wearable Chemical Hazard Detection Systems using Chip-scale Microsensors
}

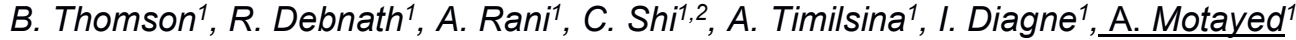 \\ ${ }^{1}$ N5 Sensors, Inc., 9610 Medical Center Dr. Suite 200, Rockville, Maryland 20852, USA, \\ 2 George Mason University, Dept. of Electrical and Computer Engineering, Fairfax, Virginia, USA \\ amotayed@n5sensors.com
}

\begin{abstract}
:
A low-power microsensor technology for rapid detection of airborne chemical threats is presented. Leveraging N5's chip-scale sensor platform, the next-generation of body-worn, i.e. wearable detectors capable of detecting various fire gases, toxic industrial chemicals, and chemical warfare agents can be realized. The sensor devices are based on a patented architecture, which combines a highperformance micro-machined semiconducting photoconductor with metal-oxide photocatalytic nanoclusters, resulting in selective micro sensors on-a-chip. The architecture represents a paradigmshift in low-power, compact chemical sensors, and addresses the capability gap that exists between the desperate need for low-power, wearable gas and chemical detectors and current inadequate mature sensor technologies that are used in handheld monitors.
\end{abstract}

Key words: gas sensors, wearable sensors, micro sensors, toxic industrial chemicals, fire gases

\section{Introduction}

Firefighters and first-responders, routinely enter environments that can contain toxic and explosive vapors, gases, and particulate matter. A firefighter may unknowingly enter an atmosphere that was thought to be void of harmful airborne products without respiratory protection and thus be at risk. A firefighter may remove or downgrade personal protection equipment prematurely, being exposed to hazardous concentrations of toxic chemicals and particulates.

Currently chemical and gas detection systems are available, however there are many problems associated with these detectors. Many are bulky and power-hungry, thus inconvenient to carry and keep charged. Sensors have drifts, cross-sensitivity to other gases, and impacted performance under challenging environmental conditions (high/low temperatures, extreme humidity). Sensors require routine calibration, frequent maintenance and replacement, activities which can be challenging to maintain. Finally, the high cost of detector systems can be prohibitive for large-scale deployment. Additionally, rapid proliferation of Industrial and commercial internet of things (IoT) and communication infrastructure has amplified the need for lowcost, wearable detector that provides seamless real-time threat monitoring for improved situational awareness.

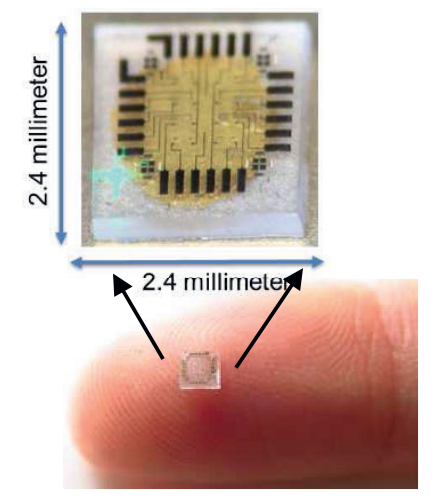

Fig. 1. An optical image of a chip containing multiple individually-addressable micro-scale gas sensors.

\section{Semiconductor-Nanophotocatalyst Hybrid Sensors}

N5's chip-scale gas sensors are essentially two-terminal photoconductors, surface functionalized with nano-scale photocatalytic material. This architecture circumvents the drawbacks of conventional metal oxide thin film gas sensors, namely high temperature operation which stems from the stability of the surface adsorbed oxygen and hydroxyl radicals at room temperature. To overcome this challenge, the engineered nanocluster layer 
works through dynamic active sites generation at the surface. The photo-desorption of adsorbed oxygen and hydroxyl radicals is utilized to improve the performance, thus reduce the operation temperature [1]. It results in low power consumption and long-term stability. Such a sensor chip with 8 individuallyaddressable microsensors, along with on-chip various calibration and compensation elements is shown in Fig. 1.

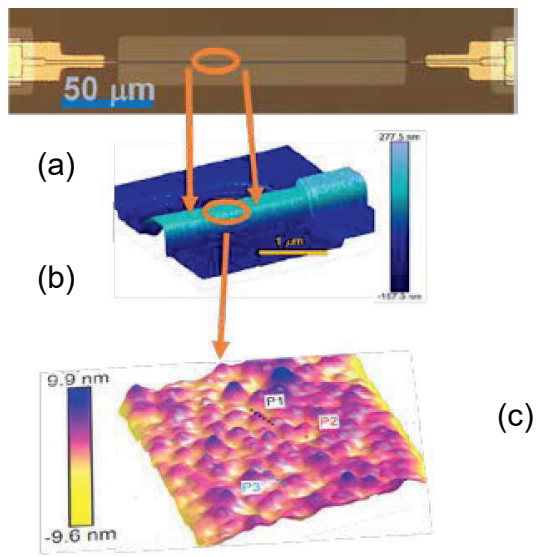

Fig. 2. (a) An optical image of a single microsensor element, (b) 3-D atomic force microscopy scan of the microsensor, (c) electrostatic force microscope image of the surface showing the nanoscale photocatalytic oxide functional layer.

A single sensor element is shown in Fig. 2a, with a trapezoidal cross-section (with top-width $\sim 250 \mathrm{~nm}$ formed by top-down etching process). The most important innovation is the patented nanophotocatalytic functionalization layer (see Fig. 2c), which acts as the target receptor, whereas the back high-quality photoconductor acts as the transducer.

\section{Manufacturing and Packaging of Chip-Scale Sensors}

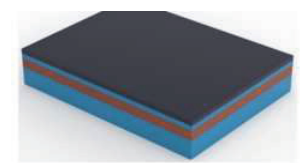

1. Wafer Planarization

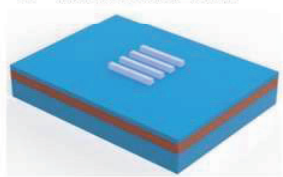

2. Pattern Development Using Project

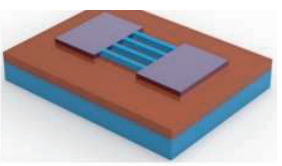

4. Contact Formation

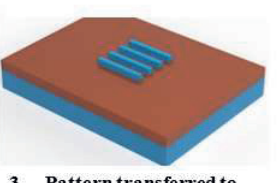

Pattern transferred to

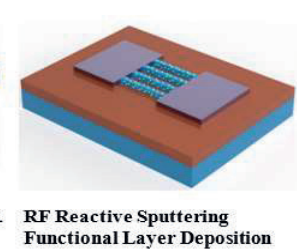

$\square$ GaN

GaN
$\square$ Passivation

$\begin{array}{ll}\square & \text { Passivation } \\ & \text { Metal Etch Stack }\end{array}$

$\square$ Contact pad

Functionalizatio

Photoresist

GaN using ICP
The actual sensor devices are manufactured using standard semiconductor manufacturing methods starting for a standard gallium nitride on silicon wafers (see Fig 3) and packaged into ceramic leadless chip carriers (see Fig. 4).

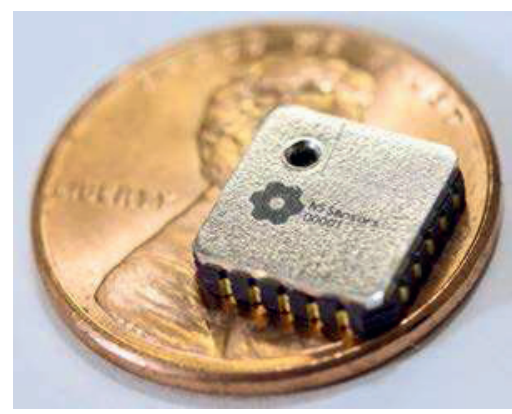

Fig. 4. Actual image of a multi-gas sensor

\section{Chemical Hazard Detection Capabilities}

Tab. 1: Current detection capabilities

\begin{tabular}{|c|c|c|}
\hline Analyte & $\begin{array}{c}\text { Limit of } \\
\text { Detection } \\
(\mathrm{ppm})\end{array}$ & $\begin{array}{c}\text { Response/Recovery } \\
\text { Time (s) }\end{array}$ \\
\hline Chlorine & 1 & $50 / 200$ \\
\hline $\begin{array}{c}\text { Hydrogen } \\
\text { Cyanide }\end{array}$ & 10 & $70 / 90$ \\
\hline $\begin{array}{c}\text { Hydrogen } \\
\text { Chloride }\end{array}$ & 10 & $60 /-$ \\
\hline Methane & 5000 & $-/-$ \\
\hline Alcohols & 50 & $50 / 100$ \\
\hline $\begin{array}{c}\text { Nitrogen } \\
\text { dioxide }\end{array}$ & 1 & $30 / 150$ \\
\hline $\begin{array}{c}\text { Sulphur } \\
\text { dioxide }\end{array}$ & 30 & $100 / 150$ \\
\hline Hydrogen & 100 & $15 / 30$ \\
\hline
\end{tabular}

\section{Future Research and Development}

One of the future goals is to develop a complete solution for chemical defense that is effective against current and future chemical threats. Such a platform technology would find its use not only in first-response, but also in homeland security, armed forces protection, and industrial safety.

\section{References}

[1] Aluri, Geetha S., et al. "GaN-nanowire/TiO 2nanocluster hybrid sensors for detection of Benzene and related aromatic compounds." Advanced Environmental, Chemical, and Biological Sensing Technologies VIII. Vol. 8024. International Society for Optics and Photonics, 2011

Fig. 3. Fabrication process flow 